gap $>\mathrm{g}:=$ SymmetricGroup $(4)$;

$\operatorname{Sym}\left(\left[\begin{array}{lll}1 & 4\end{array}\right]\right)$

gap> tbl:= CharacterTable ( $\mathrm{g}$ ); ; HasIrr ( tbl );

false

05 = total: $\begin{array}{rrrrr}1 & 4 & 13 & 14 & 4\end{array}$

gap> tblmod2:= CharacterTable( tbl, 2 );

BrauerTable( $\operatorname{Sym}([1 \ldots 4$ ] ), 2 )

1: . 2242 gap> tblmod2 = CharacterTable $($ tbl, 2 );

2: . 256 . true

Journal of Software for

01234 gap> libtbl:= CharacterTable( "M" );

$06=$ total: $1{ }^{4} 13144$ CharacterTable ( "M")

fail

gap> CharacterTable ( "Symmetric", 4 ); int a, b, c, t=11, 5, 3,0;

o6 : BettiTally 17 : 1 = betti( , Weights $\Rightarrow\{1,1\}$ )

gap $>$ ComputedBrauerTables ( tbl);

$\begin{array}{lllll}0 & 1 & 2 & 3 & 4\end{array}$

[

poly $f=x^{\wedge} a+y^{\wedge} b+z^{\wedge}(3 * c)+x^{\wedge}(c+2) * y^{\wedge}(c-1)+x^{\wedge}$ $x^{\wedge}(c-2) * y^{\wedge} c *\left(y^{\wedge} 2+t * x\right) \sim 2$;

$07=$ total: $14 \begin{array}{llll}1 & 4 & 14 & 4\end{array}$

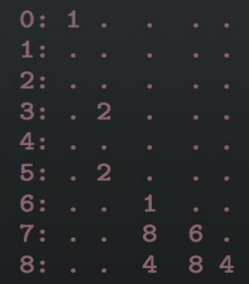

o7 : BettiTally

i8 : peek t1

$08=\operatorname{BettiTally}\{(0,\{0,0\}, 0) \Rightarrow 1\}$

$(1,\{2,2\}, 4) \Rightarrow 2$

$(1,\{3,3\}, 6) \Rightarrow 2$

$(2,\{3,7\}, 10) \Rightarrow 2$

$(2,\{4,4\}, 8) \Rightarrow 1$

$(2,\{4,5\}, 9) \Rightarrow 4$

$(2,\{5,4\}, 9) \Rightarrow 4$

(2, $\{7,3\}, 10) \Rightarrow 2$

Exterior:

$(3,\{7,4\}, 11) \Rightarrow 4$

$(4,\{5,7\}, 12) \Rightarrow 2$ over an exterior algebra

$(4,\{7,5\}, 12) \Rightarrow 2$ 


\title{
ExteriorModules: a package for computing monomial modules over an exterior algebra
}

\author{
LuCA AMATA AND MARILENA CRUPI
}

\begin{abstract}
Let $K$ be a field, $E$ the exterior algebra of a finite-dimensional $K$-vector space, and $F$ a finitely generated graded free $E$-module with homogeneous basis $g_{1}, \ldots, g_{r}$ such that $\operatorname{deg}\left(g_{1}\right) \leq$ $\operatorname{deg}\left(g_{2}\right) \leq \cdots \leq \operatorname{deg}\left(g_{r}\right)$. We present a Macaulay2 package to manage some classes of monomial submodules of $F$. The package is an extension of our ExteriorIdeals package on monomial ideals (J. of Software for Alg. and Geom. 8:7 (2018), 71-79), and contains some algorithms for computing stable, strongly stable and lexicograhic $E$-submodules of $F$. This package also includes some methods to check whether a sequence of nonnegative integers is the Hilbert function of a graded $E$-module of the form $F / M$, with $M$ a graded submodule of $F$. Moreover, if $H_{F / M}$ is the Hilbert function of a graded $E$-module $F / M$, some routines are able to compute the unique lexicograhic submodule $L$ of $F$ such that $H_{F / M}=H_{F / L}$.
\end{abstract}

1. IntRoduction. Monomial modules generalize the notion of monomial ideals which are ideals generated by monomials. Therefore, many tools in monomial ideal theory are available to deal with such a class of modules. Indeed, many statements on monomial modules can be deduced from the ones on monomial ideals. Many authors have been interested in the study of such classes of modules in different contexts and in the computation of certain invariants associated to them (see, for instance [Aramova et al. 1997; Aramova and Herzog 2000; Crupi and Ferrò 2016; Eisenbud 1995; Gasharov 1997; Herzog and Hibi 2011; Kämpf 2010; Pardue 1996]).

In this paper, we introduce a new Macaulay2 package, ExteriorModules, for manipulating special classes of monomial modules over an exterior algebra of a finite-dimensional vector space over a field. This package extends the one on monomial ideals, ExteriorIdeals, and needs it to work. An updated version of such a package is available on the Macaulay2 repository. In more detail, the package ExteriorModules provides functions to check whether a monomial module is (strongly) stable, or lexicographic, and to compute the smallest (strongly) stable module containing a given monomial module. Moreover, given $F$ a finitely generated graded free module over an exterior algebra $E$, the package allows us to characterize the Hilbert sequences of the $E$-modules of the type $F / M$, with $M$ a graded submodule of $F$.

Some service methods are inherited from ExteriorIdeals or overloaded to extend them to modules in order to optimize the implementation of the main algorithms.

MSC2020: 13A02, 15A75, 68W30.

Keywords: exterior algebra, monomial modules, Hilbert functions, algorithms.

ExteriorModules version 1.0 
2. MAthematical background. Let $K$ be a field and let $E=K\left\langle e_{1}, \ldots, e_{n}\right\rangle$ be the exterior algebra of a $K$-vector space $V$ with basis $e_{1}, \ldots, e_{n}$. For any subset $\sigma=\left\{i_{1}, \ldots, i_{d}\right\}$ of $\{1, \ldots, n\}$ with $i_{1}<i_{2}<\cdots<i_{d}$ we write $e_{\sigma}=e_{i_{1}} \wedge \cdots \wedge e_{i_{d}}=e_{i_{1}} \cdots e_{i_{d}}$, in order to simplify the notation, and call $e_{\sigma}$ a monomial of degree $d$. We set $e_{\sigma}=1$, if $\sigma=\varnothing$. The set of monomials in $E$ forms a $K$-basis of $E$ of cardinality $2^{n}$.

Let $e_{\sigma}=e_{i_{1}} \cdots e_{i_{d}} \neq 1$ be a monomial in $E$. We define

$$
\operatorname{supp}\left(e_{\sigma}\right)=\sigma=\left\{i: e_{i} \text { divides } e_{\sigma}\right\},
$$

and we write

$$
\mathrm{m}\left(e_{\sigma}\right)=\max \left\{i: i \in \operatorname{supp}\left(e_{\sigma}\right)\right\} .
$$

Moreover, we set $\mathrm{m}\left(e_{\sigma}\right)=0$, if $e_{\sigma}=1$.

From now on, we write $f g=f \wedge g$ for any two elements $f$ and $g$ in $E$. An element $f \in E$ is called homogeneous of degree $j$ if $f \in E_{j}$, where $E_{j}=\bigwedge^{j} V$. An ideal $I$ is called graded if $I$ is generated by homogeneous elements. If $I$ is graded, then $I=\bigoplus_{j \geq 0} I_{j}$, where $I_{j}$ is the $K$-vector space of all homogeneous elements $f \in I$ of degree $j$. Moreover, we use indeg $(I)$ to denote the initial degree of $I$, i.e., the minimum $s$ such that $I_{s} \neq 0$.

Let $\mathcal{M}$ be the category of finitely generated $\mathbb{Z}$-graded left and right $E$-modules $M$ such that $a m=$ $(-1)^{\operatorname{deg} a \operatorname{deg} m} m a$ for all homogeneous elements $a \in E, m \in M$.

If $M \in \mathcal{M}$, the function $H_{M}: \mathbb{Z} \rightarrow \mathbb{Z}$ given by $H_{M}(d)=\operatorname{dim}_{K} M_{d}$ is called the Hilbert function of $M$ [Bruns and Herzog 1993; Eisenbud 1995].

Let $F \in \mathcal{M}$ be a free module with homogeneous basis $g_{1}, \ldots, g_{r}$, where $\operatorname{deg}\left(g_{i}\right)=f_{i}$ for each $i=1, \ldots, r$, with $f_{1} \leq f_{2} \leq \cdots \leq f_{r}$. We write $F=\bigoplus_{i=1}^{r} E g_{i}$. The elements $e_{\sigma} g_{i}$, with $e_{\sigma}$ a monomial of $E$, are called monomials of $F$, and $\operatorname{deg}\left(e_{\sigma} g_{i}\right)=\operatorname{deg}\left(e_{\sigma}\right)+\operatorname{deg}\left(g_{i}\right)$. Furthermore, when we write $F \simeq E^{r}$, we mean that $F=\bigoplus_{i=1}^{r} E g_{i}$ is the free $E$-module with trivial homogeneous basis $g_{1}, \ldots, g_{r}$, where $g_{i}(i=1, \ldots, r)$ is the $r$-tuple having the only nonzero entry equal to 1 in the $i$-th position and such that $\operatorname{deg}\left(g_{i}\right)=0$, for all $i$.

Definition 2.1. A graded submodule $M$ of $F$ is a monomial submodule if $M$ is a submodule generated by monomials of $F$, i.e.,

$$
M=I_{1} g_{1} \oplus \cdots \oplus I_{r} g_{r},
$$

where $I_{i}$ is a monomial ideal of $E$, for each $i$.

We observe that, if $r=1$ and $f_{1}=0$ then a monomial submodule of $F$ is a monomial ideal of $E$.

Definition 2.2. A monomial ideal $I$ of $E$ is called stable if for each monomial $e_{\sigma} \in I$ and each $j<\mathrm{m}\left(e_{\sigma}\right)$ one has $e_{j} e_{\sigma \backslash\left\{\mathrm{m}\left(e_{\sigma}\right)\right\}} \in I$. I is called strongly stable if for each monomial $e_{\sigma} \in I$ and each $j \in \sigma$ one has $e_{i} e_{\sigma \backslash\{j\}} \in I$, for all $i<j$.

Remark 2.3. One can observe that the defining property of a strongly stable ideal needs to be checked only for the set of monomial generators of a monomial ideal [Amata and Crupi 2018b, Remark 2.2.]. 
Definition 2.4. A monomial submodule $M=\bigoplus_{i=1}^{r} I_{i} g_{i}$ of $F$ is called almost (strongly) stable if $I_{i}$ is a (strongly) stable ideal of $E$, for each $i$.

Definition 2.5. A monomial submodule $M=\bigoplus_{i=1}^{r} I_{i} g_{i}$ of $F$ is called (strongly) stable if $I_{i}$ is a (strongly) stable ideal of $E$, for each $i$, and $\left(e_{1}, \ldots, e_{n}\right)^{f_{i+1}-f_{i}} I_{i+1} \subseteq I_{i}$, for $i=1, \ldots, r-1$.

Given a monomial ideal $I$ of $E$, we denote by $G(I)$ the unique minimal set of monomial generators of $I$, and by $G(I)_{d}$ the set of all monomials $u \in G(I)$ such that $\operatorname{deg}(u)=d, d>0$. Similarly, for every monomial submodule $M=\bigoplus_{i=1}^{r} I_{i} g_{i}$ of $F$, we write

$$
\begin{aligned}
G(M) & =\left\{u g_{i}: u \in G\left(I_{i}\right), i=1, \ldots, r\right\}, \\
G(M)_{d} & =\left\{u g_{i}: u \in G\left(I_{i}\right)_{d-f_{i}}, i=1, \ldots, r\right\} .
\end{aligned}
$$

For the classification of the Hilbert functions of graded $E$-modules, the class of lexicographic modules plays a crucial role [Amata and Crupi 2020a; 2020b]. Moreover, such a class of monomial modules is essential if one wants to determine certain upper bounds for the graded Betti numbers of graded $E$ modules [Amata and Crupi 2018a; 2019].

Given a nonempty subset $S$ of $E$ (respectively, of $F$ ), we denote by $\operatorname{Mon}(S)$ the set of all monomials in $S$ (respectively, in $F$ ). Moreover, we denote by $\operatorname{Mon}_{d}(S)$ the set of all monomials of degree $d$ in $S$.

We denote by $>_{\text {lex }}$ the lexicographic order (lex order, for short) on $\operatorname{Mon}_{d}(E)$, i.e., if $e_{\sigma}=e_{i_{1}} e_{i_{2}} \cdots e_{i_{d}}$ and $e_{\tau}=e_{j_{1}} e_{j_{2}} \cdots e_{j_{d}}$ are monomials belonging to $\operatorname{Mon}_{d}(E)$ with $1 \leq i_{1}<i_{2}<\cdots<i_{d} \leq n$ and $1 \leq j_{1}<j_{2}<\cdots<j_{d} \leq n$, then $e_{\sigma}>_{\operatorname{lex}} e_{\tau}$ if $i_{1}=j_{1}, \ldots, i_{s-1}=j_{s-1}$ and $i_{s}<j_{s}$ for some $1 \leq s \leq d$.

Definition 2.6. Let $L$ be a nonempty subset of $\operatorname{Mon}_{d}(E)$. $L$ is called a lexicographic segment (lex segment, for short) of degree $d$ if for all $v \in L$ and all $u \in \operatorname{Mon}_{d}(E)$ such that $u>_{\text {lex }} v$, we have that $u \in L$.

Definition 2.7. Let $I$ be a monomial ideal of $E$. $I$ is called a lexicographic ideal (lex ideal, for short) if for all monomials $v \in I$ and all monomials $u \in E$ with $\operatorname{deg} v=\operatorname{deg} u$ and $u>_{\text {lex }} v$, then $u \in I$, i.e., $\operatorname{Mon}_{d}(I)$ is a lex segment, for all $d$.

Remark 2.8. The trivial ideals of $E$, i.e., (0) and $E$ itself, are considered monomial lex ideals.

Now, we extend the previous definitions to monomial submodules of $F$. To do this, we order the set of monomials $\operatorname{Mon}(F)$ by using the ordering $>_{\operatorname{lex}_{F}}$ defined as follows: if $u g_{i}$ and $v g_{j}$ are monomials of $F$ such that $\operatorname{deg}\left(u g_{i}\right)=\operatorname{deg}\left(v g_{j}\right)$, then $u g_{i}>_{\operatorname{lex}_{F}} v g_{j}$ if $i<j$ or $i=j$ and $u>_{\text {lex }} v$.

Definition 2.9. Let $N$ be a nonempty subset of $\operatorname{Mon}_{d}(F) . N$ is called a lexicographic segment of $F$ (lex $F$ segment, for short) of degree $d$ if for all $v \in N$ and all $u \in \operatorname{Mon}_{d}(F)$ such that $u>_{\operatorname{lex}_{F}} v$, then $u \in N$.

Definition 2.10. Let $L$ be a monomial submodule of $F$. $L$ is a lex submodule if for all $u, v \in \operatorname{Mon}_{d}(F)$ with $v \in L$ and $u>\operatorname{lex}_{F} v$, one has $u \in L$, for every $d$, i.e., $\operatorname{Mon}_{d}(L)$ is a lex $F \operatorname{segment}_{\text {of }} \operatorname{degree} d$, for each degree $d$. 
An equivalent definition of a lex submodule is the following one [Amata and Crupi 2018a, Proposition 3.12] (see also [Crupi and Ferrò 2016, Proposition 3.8]).

Definition 2.11. Let $L$ be a graded submodule of $F$. $L$ is a lex submodule of $F$ if $L=\bigoplus_{i=1}^{r} I_{i} g_{i}$, with $I_{i}$ lex ideals of $E(i=1, \ldots, r)$, and $\left(e_{1}, \ldots, e_{n}\right)^{\rho_{i}+f_{i}-f_{i-1}} \subseteq I_{i-1}$, for $i=2, \ldots, r$, with $\rho_{i}=\operatorname{indeg} I_{i}$.

Remark 2.12. The class of lex submodules of $F$ is obviously contained in the class of strongly stable submodules, and consequently in the class of the stable ones.

A particular overclass of the class of lex submodules, introduced in [Amata and Crupi 2018a] for bounding the graded Betti numbers of a graded $E$-module, is the following one.

Definition 2.13. Let $L$ be a graded submodule of $F$. $L$ is an almost lexicographic submodule if $L=$ $\bigoplus_{i=1}^{r} I_{i} g_{i}$, with $I_{i}$ lex ideals of $E(i=1, \ldots, r)$.

In order to discuss the Hilbert functions of quotients of free $E$-modules, we need some notation and remarks. For more details on the subject see [Amata and Crupi 2020a; 2020b].

Firstly, we set

$$
\left(\begin{array}{l}
m \\
k
\end{array}\right)=0 \quad \text { if } m<k \text { or } k<0
$$

One can observe that if $F=\bigoplus_{i=1}^{r} E g_{i}$, deg $g_{i}=f_{i}$, for $i=1, \ldots, r$ and $f_{1} \leq \cdots \leq f_{r}$, we have that

$$
H_{F}(d)=\sum_{i=1}^{r} H_{E g_{i}}(d)=\sum_{i=1}^{r}\left(\begin{array}{c}
n \\
d-f_{i}
\end{array}\right) .
$$

Hence, if $M$ is a graded submodule of $F$, one has

$$
H_{F / M}(d)+H_{M}(d)=\sum_{i=1}^{r}\left(\begin{array}{c}
n \\
d-f_{i}
\end{array}\right),
$$

where $\left(\begin{array}{c}n \\ d-f_{i}\end{array}\right)$ is the number of monomials of degree $d-f_{i}$ in $E$.

As a consequence, we have that

$$
H_{F}(d)=\operatorname{dim}_{K} F_{d}=0, \quad \text { for } d<f_{1} \text { and } d>f_{r}+n .
$$

If $M$ is a monomial submodule of $F$, from (1), it follows that

$$
H_{F / M}(t)=\sum_{i=f_{1}}^{f_{r}+n} H_{F / M}(i) t^{i},
$$

and we can associate to $F / M$ the sequence

$$
\left(H_{F / M}\left(f_{1}\right), H_{F / M}\left(f_{1}+1\right), \ldots, H_{F / M}\left(f_{r}+n\right)\right) \in \mathbb{N}_{0}^{f_{r}+n-f_{1}+1} .
$$

Such a sequence is called the Hilbert sequence of $F / M$, and we denote it by $H s_{F / M}$. The integers $f_{1}, f_{1}+1, \ldots, f_{r}+n$ are called the $H s_{F / M}$-degrees. 
Let us define

$$
\text { indeg } H s_{F / M}=\min \left\{d: H_{F / M}(d) \neq 0\right\}, \quad \text { for } d=f_{1}, \ldots, f_{r}+n \text {. }
$$

We use the notation $[p]$ for the set $\{1,2, \ldots, p\}$.

The entries $H_{F / M}\left(f_{i}\right)(i=1, \ldots, r)$ are called the critical values of $H s_{F / M}$. Moreover, the integers

$$
\mu_{f_{i}}=\left|\left\{s \in[r]: f_{s}=f_{i}\right\}\right|, \quad \text { for } i=1,2, \ldots, r
$$

are called the multiplicity of $H_{F / M}\left(f_{i}\right)$.

We can observe that some critical values can be zero, and this implies that all the entries of the Hilbert sequence are zero until the next nonzero critical value. So it makes sense to investigate the minimum critical value of a Hilbert sequence to get important information about the behavior of a Hilbert function.

Let $k$ be the minimum integer such that $H_{F / M}\left(f_{k}\right) \neq 0$, i.e., indeg $H s_{F / M}=f_{k}$. The integer $H_{F / M}\left(f_{k}\right)$ is called the initial critical value (of $F / M$ ) and $f_{k}$ the initial critical degree (of $F / M$ ). Moreover, we have that

$$
H_{F / M}\left(f_{k}\right) \leq \mu_{f_{k}}
$$

and

$$
H_{F / M}\left(f_{k}+1\right) \leq n \mu_{f_{k}}+\mu_{f_{k}+1} \text {. }
$$

Now we have all the necessary ingredients to quote the main result on the classification of Hilbert functions of quotients of graded free $E$-modules [Amata and Crupi 2020a, Theorem 4.2]. The pivotal idea of such a classification is that if $M$ is a graded submodule of $F$, then there exists a unique lex submodule of $F$ with the same Hilbert function as $M$.

Let $a$ and $i$ be two positive integers. Then $a$ has the unique $i$-th Macaulay expansion [Herzog and Hibi 2011, Lemma 6.3.4]

$$
a=\left(\begin{array}{c}
a_{i} \\
i
\end{array}\right)+\left(\begin{array}{c}
a_{i-1} \\
i-1
\end{array}\right)+\cdots+\left(\begin{array}{c}
a_{j} \\
j
\end{array}\right)
$$

with $a_{i}>a_{i-1}>\cdots>a_{j} \geq j \geq 1$. We define

$$
a^{(i)}=\left(\begin{array}{c}
a_{i} \\
i+1
\end{array}\right)+\left(\begin{array}{c}
a_{i-1} \\
i
\end{array}\right)+\cdots+\left(\begin{array}{c}
a_{j} \\
j+1
\end{array}\right) .
$$

We also set $0^{(i)}=0$ for all $i \geq 1$.

For $p, q \in \mathbb{Z}$ with $p<q$, let us define the set

$$
[p, q]=\{j \in \mathbb{Z}: p \leq j \leq q\}
$$

Theorem 2.14 [Amata and Crupi 2020a, Theorem 4.2]. Let $\left(f_{1}, f_{2}, \ldots, f_{r}\right) \in \mathbb{Z}^{r}$ be an $r$-tuple such that $f_{1} \leq f_{2} \leq \cdots \leq f_{r}$, and let $\left(h_{f_{1}}, h_{f_{1}+1}, \ldots, h_{f_{r}+n}\right)$ be a sequence of nonnegative integers. Set

$$
s=\min \left\{k \in\left[f_{1}, f_{r}+n\right]: h_{k} \neq 0\right\}
$$


and

$$
\tilde{r}_{j}=\left|\left\{p \in[r]: f_{p}=s+j\right\}\right|, \quad \text { for } j=0,1 .
$$

Then the following conditions are equivalent:

(a) $\sum_{i=s}^{f_{r}+n} h_{i} t^{i}$ is the Hilbert series of a graded $E$-module $F / M$, with $F=\bigoplus_{i=1}^{r} E g_{i}$ a finitely generated graded free E-module with the basis elements $g_{i}$ of degrees $f_{i}$.

(b) $h_{s} \leq \tilde{r}_{0}, h_{s+1} \leq n \tilde{r}_{0}+\tilde{r}_{1}, h_{i}=\sum_{j=N+1}^{r}\left(\begin{array}{c}n \\ i-f_{j}\end{array}\right)+a$, where a is a positive integer less than $\left(\begin{array}{c}n \\ i-f_{N}\end{array}\right)$, $0<N \leq r$, and $h_{i+1} \leq \sum_{j=N+1}^{r}\left(\begin{array}{c}n \\ i-f_{j}+1\end{array}\right)+a^{\left(i-f_{N}\right)}, i=s+1, \ldots, f_{r}+n$.

(c) There exists a unique lex submodule L of a finitely generated graded free E-module $F=\bigoplus_{i=1}^{r} E g_{i}$ with the basis elements $g_{i}$ of degrees $f_{i}$, and such that $\sum_{i=s}^{f_{r}+n} h_{i} t^{i}$ is the Hilbert series of $F / L$.

From now on, if $M$ is a monomial submodule of the finitely generated graded free $E$-module $F=$ $\bigoplus_{i=1}^{r} E g_{i}$, we denote by $M^{\text {lex }}$ the unique lex submodule of $F$ with the same Hilbert function as $M$. $M^{\text {lex }}$ is called the lex submodule associated to $M$.

Remark 2.15. Theorem 2.14 generalizes the well-known Kruskal-Katona's theorem in [Aramova et al. 1997], and can be also obtained via results on ideals in an exterior algebra [Amata and Crupi 2020b, Criterion 3.3]. The underlying algorithm is implemented as the lexModuleBySequences method in the ExteriorModules package.

3. EXAMPLES. In this section, we collect some examples in order to describe the algorithms.

In what follows, let $F=\bigoplus_{i=1}^{r} E g_{i}$ be a finitely generated graded free $E$-module such that

$$
\operatorname{deg}\left(g_{1}\right) \leq \operatorname{deg}\left(g_{2}\right) \leq \cdots \leq \operatorname{deg}\left(g_{r}\right)
$$

Example 3.1. Let $M$ be a monomial submodule of the graded free module $F$, we illustrate functions from the ExteriorModules package (analogous to those for ideals [Amata and Crupi 2018b]) in order to check whether $M$ is (strongly) stable or (almost) lex, and to produce the smallest (strongly) stable submodule containing $M$.

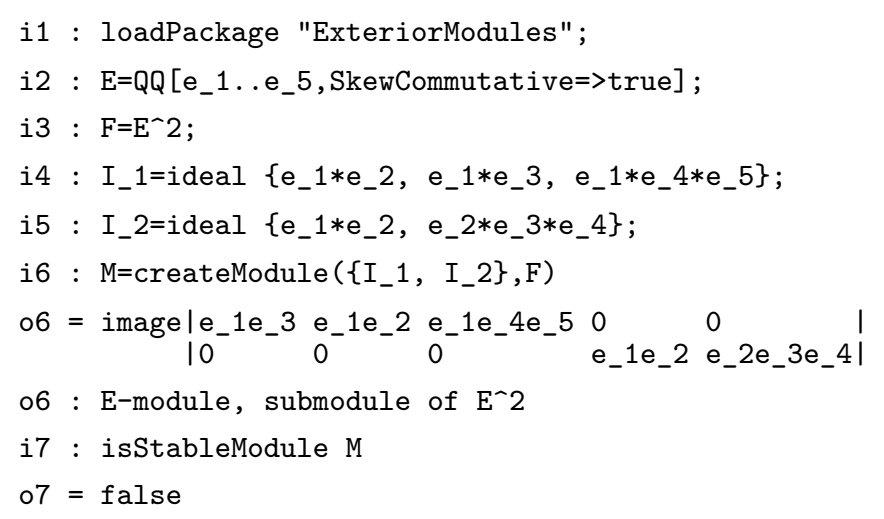

The submodule $M$ is almost stable but not stable. In fact, the monomial $e_{2} e_{3} e_{4}$ does not belong to $I_{1}$ 
(Definition 2.5). We can compute the smallest stable submodule of $F$ containing $M$ by the function stableModule (module).

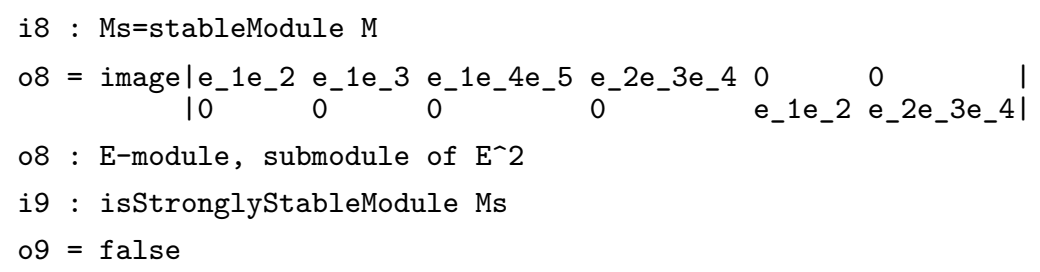

The submodule $M s$ is stable, and but neither almost strongly stable nor strongly stable. In fact, the ideal $\left(e_{1} e_{2}, e_{2} e_{3} e_{4}\right)$ is not strongly stable. We compute the smallest strongly stable submodule of $F$ containing $M s$ by using the function stronglyStableModule (module):

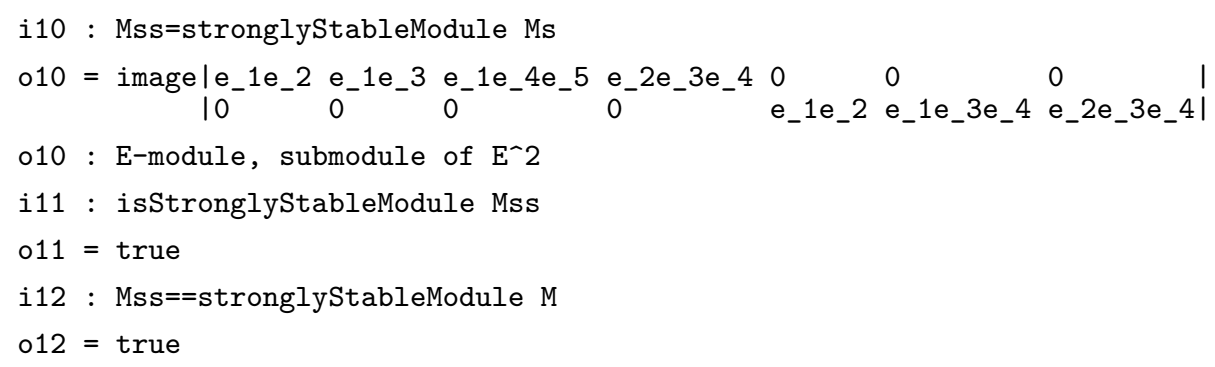

The submodule $M s s$ is not an almost lex submodule of $F$. Indeed, the ideal $\left(e_{1} e_{3} e_{4}, e_{2} e_{3} e_{4}\right)$ is not lex.

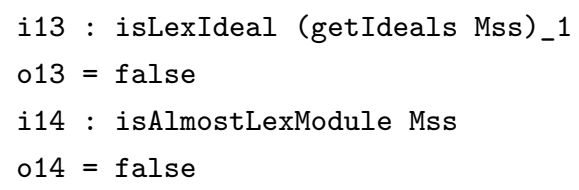

Remark 3.2. The functions stableModule (module) and stronglyStableModule (module) allow the construction of (strongly) stable submodules of a finitely generated graded free module $F$. The methods to compute the smallest stable and strongly stable submodule containing a given submodule are useful, although they do not preserve invariants. In fact, the computation by hand of a stable or a strongly stable submodule implies some tedious calculations overall in the case when the elements of the homogeneous basis of $F$ have different degrees. Furthermore, it is worth pointing out that such methods are analogous to the Macaulay2 function borel that computes the smallest borel ideal containing a given ideal.

Example 3.3. Let $h$ be a sequence of nonnegative integers. We describe how one can check whether $h$ is a Hilbert sequence of a graded $E$-module of the type $F / M$, with $M$ graded submodule of $F$. The key tools are the functions lexModuleBySequences (list,F) (Remark 2.15), isHilbertSequence (list,F), and lexModule (list,F). The first function verifies if a list of nonnegative integers of a given length is a Hilbert function; the other ones return a lex submodule of $F$ if and only if the list is a Hilbert sequence. In more detail, if $h s$ is a given Hilbert sequence, the lex submodule of $F$ produced by both the functions lexModule $(h s, F)$ and lexModuleBySequences $(h s, F)$ is the unique lex submodule $L$ of $F$ with $H_{F / L}=h s$. These functions work also in the case when the basis elements of the free module $F$ have 
different degrees.

i1 : loadPackage "ExteriorModules";

i2: $E=Q Q\left[e \_1 . . e \_4\right.$, SkewCommutative=>true] ;

i3: $F=E^{\wedge} 3$;

i4: $\mathrm{hs}=\{3,12,16,6,0\}$;

i5 : lexModule (hs, F)

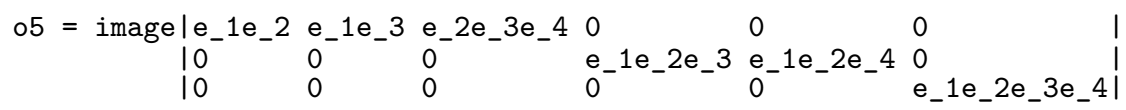

o5 : E-module, submodule of $\mathrm{E}^{\wedge} 3$

i6: $F=E^{\wedge}\{2,0,-2\}$;

i7 : hs $=\{1,4,5,4,6,5,6,3,0\}$;

i8 : lexModuleBySequences (hs, F)

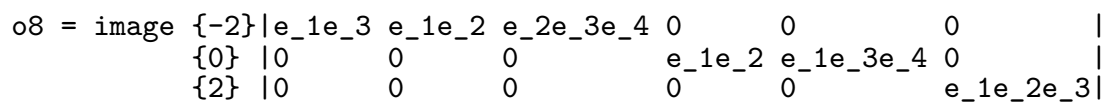

o8 : E-module, submodule of $\mathrm{E}^{\wedge} 3$

i9: $F=E^{\wedge}\{3,1,-2\}$;

i10: $\mathrm{hs}=\{1,2,2,4,3,3,4,5,2,0\}$;

i11 : isHilbertSequence (hs, F)

$011=$ false

Example 3.4. Given a graded submodule $M$ of $F$, we illustrate another way for computing the unique lex submodule associated to $M$. Given $M$, we compute $M^{\text {lex }}$ by the function lexModule (module). The procedure for the computation of the required lex submodule is based on the constructive proof of Theorem 2.14, (b) $\Rightarrow$ (c).

i1 : loadPackage "ExteriorModules";

i2 : $E=Q Q\left[e_{-} 1 \ldots e_{-} 4\right.$, SkewCommutative=>true];

i3 : $F=E^{\wedge} 3$;

i4 : I_1=ideal $\left\{e_{-} 1, e_{-} 2 * e_{-} 3 * e_{-} 4\right\}$;

i5 : I_2=ideal $\left\{e_{-} 1 * e_{-} 2, e_{-} 1 * e_{-} 3 * e_{-} 4\right\}$;

i6 : I_3=ideal $\left\{e_{-} 1 * e_{-} 2 * e_{-} 3\right\}$;

i7 : M=createModule $\left(\left\{I_{-} 1, I_{-} 2, I_{-} 3\right\}, F\right)$

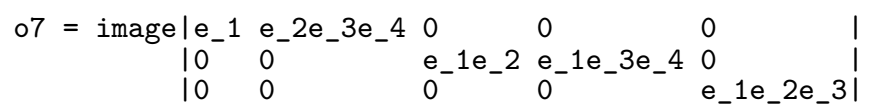

o7 : E-module, submodule of $\mathrm{E}^{\wedge} 3$

i8 : isAlmostLexModule M

$08=$ true

i9 : isLexModule M

$09=$ false

i10 : L=lexModule $M$

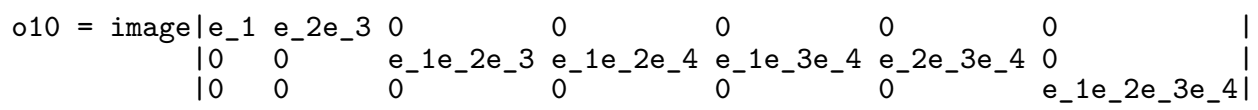

o10 : E-module, submodule of E`3 


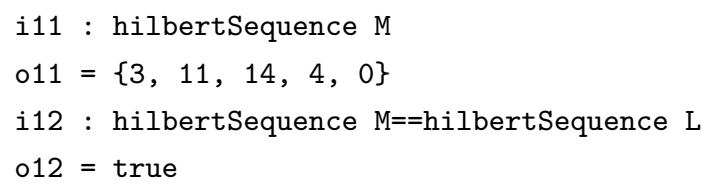

The function lexModule (module) also works in the case when the basis elements of the free module $F$ have different degrees.

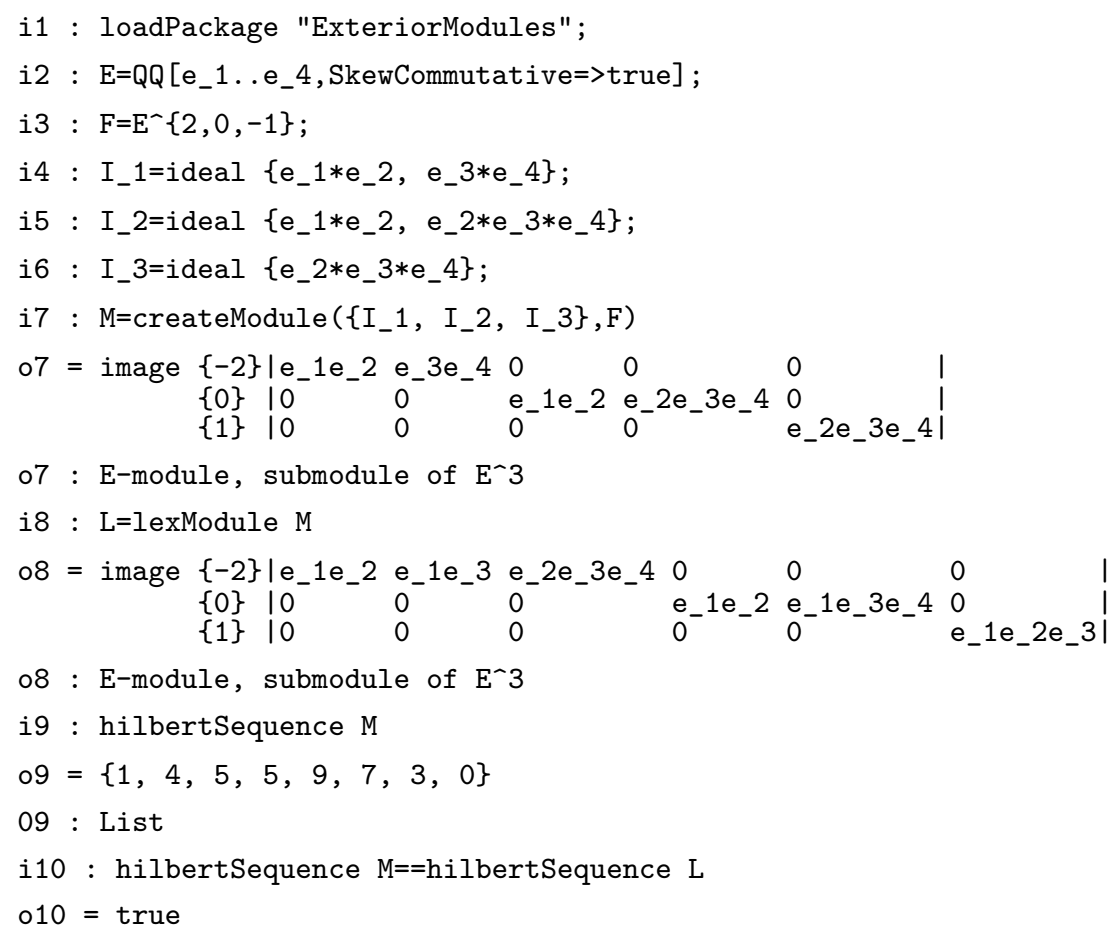

4. CONCLUSIONS AND PERSPECTIVES. The procedures described in this paper are part of the Macaulay2 package ExteriorModules (which uses the ExteriorIdeals package [Amata and Crupi 2018b]), and tested with Macaulay 1.14 as well as all the examples in this paper.

As far as we know, specific packages for manipulating classes of monomial modules over an exterior algebra have not been implemented yet. Many characterizations and algorithmic methods presented in the package are due to the authors of this paper. We believe that these packages may reveal useful further applications. Indeed, it would be nice to create functions for the computation of the generic initial module of a graded $E$-module $M$ in the category $\mathcal{M}$, which is a strongly stable module with the same Hilbert function as $M$.

Moreover, it would be interesting to manage the dual module of a graded $E$-module $M \in \mathcal{M}$ in a general case, i.e., when $M$ is a submodule of a finitely generated graded free module whose basis elements have different degrees. The case when the basis elements have the same degree was faced and solved in [Amata and Crupi 2019].

These problems are currently under investigation by the authors. 


\section{APPENDIX: LIST OF FUNCTIONS PROVIDED.}

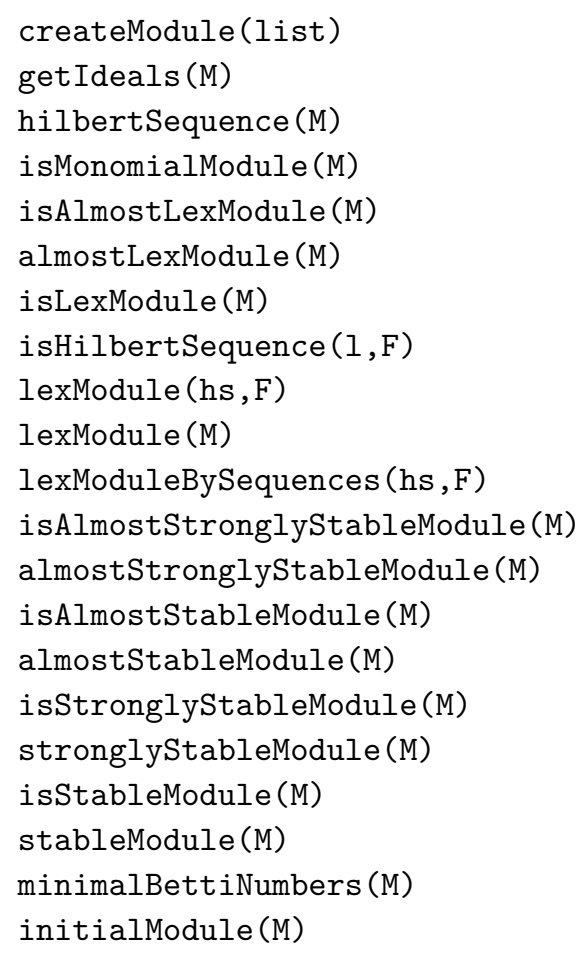

Give the monomial module from a list of ideals

Get ideals from a monomial module $\mathrm{M}$

Give the Hilbert function sequence of $M$

Check whether a module M is monomial

Check whether a module $M$ is almost lex

Give the almost lex module associated to M

Check whether a module $M$ is lex

Check whether the Kruskal-Katona theorem is satisfied for 1

Give the lex module with the given Hilbert sequence hs

Give the lex module associated to M

Give the lex module with the given Hilbert function 2.15

Check whether a module $\mathrm{M}$ is almost strongly stable

Give the minimal almost strongly stable module containing M

Check whether a module $\mathrm{M}$ is almost stable

Give the minimal almost stable module containing $\mathrm{M}$

Check whether a module $M$ is strongly stable

Give the minimal strongly stable module containing M

Check whether a module $M$ is stable

Give the minimal stable module containing $M$

Give the (minimal) Betti numbers of $\mathrm{M}$

Give the initial module of $M$

SuPPLEMENT. The online supplement contains version 1.0 of ExteriorModules.

\section{REFERENCES.}

[Amata and Crupi 2018a] L. Amata and M. Crupi, "Bounds for the Betti numbers of graded modules with given Hilbert function in an exterior algebra via lexicographic modules", Bull. Math. Soc. Sci. Math. Roumanie (N.S.) 61(109):3 (2018), 237-253. MR Zbl

[Amata and Crupi 2018b] L. Amata and M. Crupi, "ExteriorIdeals: A package for computing monomial ideals in an exterior algebra", J. of Software for Alg. and Geom. 8:7 (2018), 71-79. Zbl

[Amata and Crupi 2019] L. Amata and M. Crupi, "Minimal resolutions of graded modules over an exterior algebra", Atti Accad. Peloritana Pericolanti Cl. Sci. Fis. Mat. Natur. 97:1 (2019), art. id. A5. MR Zbl

[Amata and Crupi 2020a] L. Amata and M. Crupi, "A generalization of Kruskal-Katona's theorem”, An. Ştiinţ. Univ. "Ovidius" Constanţa Ser. Mat. 28:2 (2020), 35-52. MR

[Amata and Crupi 2020b] L. Amata and M. Crupi, "Hilbert functions of graded modules over an exterior algebra: an algorithmic approach”, Int. Electron. J. Algebra 27 (2020), 271-287. MR Zbl

[Aramova and Herzog 2000] A. Aramova and J. Herzog, "Almost regular sequences and Betti numbers", Amer. J. Math. 122:4 (2000), 689-719. MR Zbl

[Aramova et al. 1997] A. Aramova, J. Herzog, and T. Hibi, "Gotzmann theorems for exterior algebras and combinatorics", $J$. Algebra 191:1 (1997), 174-211. MR Zbl

[Bruns and Herzog 1993] W. Bruns and J. Herzog, Cohen-Macaulay rings, Cambridge Studies in Advanced Mathematics 39, Cambridge University Press, 1993. MR

[Crupi and Ferrò 2016] M. Crupi and C. Ferrò, "Squarefree monomial modules and extremal Betti numbers", Algebra Colloq. 23:3 (2016), 519-530. MR Zbl 
Amata and Crupi

[Eisenbud 1995] D. Eisenbud, Commutative algebra: with a view toward algebraic geometry, Graduate Texts in Mathematics 150, Springer, 1995. MR Zbl

[Gasharov 1997] V. Gasharov, "Extremal properties of Hilbert functions”, Illinois J. Math. 41:4 (1997), 612-629. MR Zbl

[Herzog and Hibi 2011] J. Herzog and T. Hibi, Monomial ideals, Graduate Texts in Mathematics 260, Springer, 2011. MR $\mathrm{Zbl}$

[Kämpf 2010] G. Kämpf, Module theory over the exterior algebra with applications to combinatorics, Ph.D. thesis, Osnabrück, 2010.

[Macaulay2] D. R. Grayson and M. E. Stillman, "Macaulay2: a software system for research in algebraic geometry", available at http://www.math.uiuc.edu/Macaulay2.

[Pardue 1996] K. Pardue, "Deformation classes of graded modules and maximal Betti numbers", Illinois J. Math. 40:4 (1996), 564-585. MR Zbl

RECEIVED: 16 Jun 2020

REVISED: 5 May 2021

ACCEPTED: 3 Jun 2021

\section{LUCA AMATA:}

lamata@unime.it

Department of Mathematics and Computer Sciences, Physical and Earth Sciences, University of Messina, Messina, Italy

\section{MARILENA CRUPI:}

mcrupi@unime.it

Department of Mathematics and Computer Sciences, Physical and Earth Sciences, University of Messina, Messina, Italy 

Phylogenetic trees

Hector Baños, Nathaniel Bushek, Ruth Davidson, Elizabeth Gross, Pamela E.

Harris, Robert Krone, Colby Long, Allen Stewart and Robert Walker

Software for doing computations in graded Lie algebras

Clas Löfwall and Samuel Lundqvist

The relative canonical resolution: Macaulay2-package, experiments and conjectures

Christian Bopp and Michael Hoff

The FrobeniusThresholds package for Macaulay2

Daniel J. Hernández, Karl Schwede, Pedro Teixeira and Emily E. Witt

Computing theta functions with Julia

Daniele Agostini and Lynn Chua

Decomposable sparse polynomial systems

Taylor Brysiewicz, Jose Israel Rodriguez, Frank Sottile and Thomas Yahl

A package for computations with sparse resultants

Giovanni Staglianò

ExteriorModules: a package for computing monomial modules over an exterior algebra

Luca Amata and Marilena Crupi

The Schur-Veronese package in Macaulay2

Juliette Bruce, Daniel Erman, Steve Goldstein and Jay Yang

admcycles - a Sage package for calculations in the tautological ring of the moduli space of stable curves

Vincent Delecroix, Johannes Schmitt and Jason van Zelm

Coding theory package for Macaulay2

Taylor Ball, Eduardo Camps, Henry Chimal-Dzul, Delio Jaramillo-Velez, Hiram

López, Nathan Nichols, Matthew Perkins, Ivan Soprunov, German Vera-Martínez and Gwyn Whieldon

Threaded Gröbner bases: a Macaulay2 package

Sonja Petrović and Shahrzad Zelenberg

Standard pairs of monomial ideals over nonnormal affine semigroups in SageMath Byeongsu Yu

Computations with rational maps between multi-projective varieties Giovanni Staglianò 